\title{
Influence of Defects on the Splitting of the Acceptor Ground State in Silicon
}

\section{A. Ambrosy and $K$. Laßmann}

Physikalisches Institut der Universität Stuttgart, Pfaffenwaldring 57 D-7000 Stuttgart 80 , Fed. Rep. of Germany

A.M. de Goër and B. Salce

Centre d'Etudes Nucleaires de Grenoble, F-38000 Grenoble, France

H. Zeile

Valvo Röhren-und Halbleiterwerke der Philips GmbH, Stresemannallee 101 D-2000 Hamburg 54, Fed Rep. of Germany

The distribution of strain fields from specified defects in otherwise pure silicon crystals is reflected in the resulting distribution of splittings $E$ of the partially orbitally degenerate $\Gamma_{8}$ ground state of effective mass acceptors. The spectral density $N(E)^{8}$ can be probed by resonant scattering of $h_{v}=E$ ultrasonic [1] or $3.8 \mathrm{kT} \cong \mathrm{E}$ thermal phonons [2]. The results of both methods (in the following: $\alpha(v)$ and $\kappa(T))$ are compared for $S i(B)$ and $S i(I n)$ crystals containing definite amounts of $\mathrm{C}$ and 0 [Tab.1]. Monte Carlo calculations (M.C.) for point defects and for $60^{\circ}$ dislocations in Si (isotropic approximation) have been made to obtain $N(E)$ as well as $D_{1}(E)$, the latter being the mean coupling constant of a specified phonon ( $L, T 1, T 2,[100],[110],[111])$ to splittings at $E$. This average coupling for all 9 types of phonons practically does not depend on $E$ and therefore presumably also for the thermal phonons. Thus, though in both experiments $D^{2}(E) \cdot N(E)$ is measured the form of $N(E)$ is preserved and $D$ can be estimated by integrating over the whole distribution normaltzing with $n a=\int N(E) d E$. The calculated distribution for point defects derives from Lorentzians and from Gaussians for dislocations. Fig.l shows $D^{2} \cdot N(E) / n_{a}$ for $S i(B)$ crystals with various concentrations of point defects from $\alpha(\nu)$. Analysis shows that only for the crystal with the highest concentration of point defects (S 80 ) $D^{2} \cdot N(E) / n_{a}$ can be fitted by the calculated "Lorentzian". The smaller the concentration the more "Gaussian" is mixed into N(E) merging into the quasi Gaussian residual distribution of unknown origin of the pure crystal \$ 87 . \$125 and S 54 of Fig.2
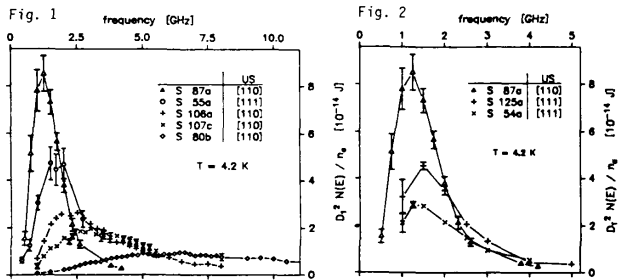

Fig.1. Splitting distribution of $\operatorname{Si}(B)$ with point defects

Fig.2. Splitting distribution of pure $S i$ for different concentration of boron 


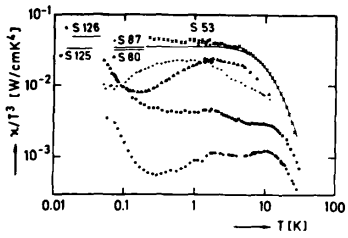

Fig.3. Themal scattering in $\overline{\mathrm{Si}(\mathrm{B})}$

are pure crystals with high $[B]$, where a dynamic $B-B$ interaction had been found by hole burning [3]. Possibly an interaction shows up also in these curves, not so much in the form of $N(E)$ but in a reduction of $D_{1}$. Analogous results in the form $\times(T) / T^{3}$ vs. $T$ are shown for crystals with as sawn side faces in Fig.3. The extra scattering below $1 \mathrm{~K}$ is due to the split levels, whereas the depression around $5 \mathrm{~K}$ - going monotonously with [B] - is interpreted as due to Jahn-Teller resonance scattering [4]. Boundary scattering for the defect-free and undoped $S 53 \mathrm{c}$ is found to be temperature dependent and about $20 \%$ larger than the theoretical Casimir term. A check with chemically polished side faces and also with an extra dummy collar showed that there is only negligible change for the $S 80$ but that thermometer mounting and strains from side face damage may have a significant influence for $S 87$ with narrow distribution. For the $S 53$ with polished side faces $\mathrm{K}(\mathrm{T}) / \mathrm{T}^{3}$ increases by about 1.6 at $2 \mathrm{~K}$ to 3.4 at $60 \mathrm{mK}$. These facts make it difficult to analyse narrow distributions of small acceptor concentrations were the extra scattering is at low temperatures and weak. In crystals with high [B] (S 125,S 126) the scattering regime is much larger than expected from $\alpha(v)$. Again the $S 80$ can be fitted by the calculated distribution but with a maximum at $8.3 \mathrm{GHz}$ instead of about $6.5 \mathrm{GHz}$ as obtained from $\alpha(v)$. The experimental/theoretical average coupling constants are $1.22 / 1.2 \mathrm{eV}$ and $1.94 / 2.22 \mathrm{eV}$ for $k$ (T) and $\alpha(v)$ respectively.

Table 1: $\mathrm{Si}(\mathrm{B})$ samples

\begin{tabular}{|c|c|c|c|c|c|c|}
\hline somple & thcm] & $\begin{array}{c}\text { concent } \\
\text { [B] }\end{array}$ & $\begin{array}{l}\text { otions [1 } \\
\text { [0] }\end{array}$ & $\begin{array}{l}\left.\mathrm{O}^{21} \mathrm{~m}^{-3}\right] \\
\text { [C] }\end{array}$ & $\begin{array}{c}u_{\text {max }} \text { in } \\
\text { (v) }\end{array}$ & $\begin{array}{l}\mathrm{Hz} \text { from } \\
\text { (dT) }\end{array}$ \\
\hline $\begin{array}{lc}\text { S } & 53^{\circ} \\
\text { S } & 54^{\circ} \\
\text { S } & 55 \\
\text { S } & 80 \\
\text { S } & 87^{\circ} \\
\text { S } & 106 \\
\text { S } & 107 \\
\text { S } & 125^{\circ} \\
\text { S } & 126\end{array}$ & \begin{tabular}{|c|}
$>2500.0$ \\
0.3 \\
1.6 \\
10.85 \\
2.5 \\
\\
\\
0.74 \\
0.3
\end{tabular} & $\begin{array}{c}0.005 \\
85.0 \\
9.0 \\
1.2 \\
5.4 \\
5.0 \\
1.8 \\
24.0 \\
85.0\end{array}$ & $\begin{array}{l}<0.5 \\
<2.0 \\
570.0 \\
<2.0 \\
<20.0 \\
<20.0 \\
200.0\end{array}$ & $\begin{array}{r}- \\
75.0 \\
41.0 \\
<5.0 \\
200.0 \\
280.0 \\
<50.0\end{array}$ & $\begin{array}{l}1.2 \\
1.7 \\
6.5 \\
1.2 \\
2.2 \\
2.8 \\
1.2\end{array}$ & $\begin{array}{l}8.3 \\
4.6\end{array}$ \\
\hline
\end{tabular}

- Wocker chemitronic, waso quality

For Si(In) the deformation potential constants being about 0.6 of those of $\mathrm{Si}(\mathrm{B})$, one would expect the distributions and the phonon scattering to be correspondingly smaller. A possible In-In interaction should set in at a concentration about 8 times larger than for $B$ be- 
cause of the smaller Bohr radius. In Tab. 2 reliable figures for the concentration can be given only for samples $S 52$ and $S 123$ where temperat ure-dependent $\mathrm{Hall}$ measurements have been made [5]. The results of $k(T)$ are normalized to the pure case to show the additional scattering [ $\mathrm{Fig.4}]$. The Jahn-Teller resonance at about $20 \mathrm{~K}$ increases monotonously with [In] whereas the scattering at low temperatures is due to the splitting distributions. For $S 123$ from $\alpha(v)$ we get $E_{\max }=$ $3.2 \mathrm{GHz}$ as compared to $\sim 5 \mathrm{GHz}$ from $k(\mathrm{I})$. Estimating the elastic strength $A$ from the covalent radii gives $A_{I n} / A C=0.68$ and taking into account the deformation potential constants one would obtain $E_{\max }=0.5$ GHz from M.C. For the S 52 with high concentration of point defects a fit somewhat broader than "Lorentzian" gives $E_{\text {max }}=14.5 \mathrm{GHz}$, whereas from M.C. one would expect $E_{\text {max }} \leqslant 9 \mathrm{GHz}$ if the indicated figures for [In] and [0] are correct. The deformation potential constant from this analysis is $0.24 \mathrm{eV}$ as compared to the theoretical value $0.72 \mathrm{eV}$. Since [In] is rather high the reduction could be due to an In-In interaction in analogy to the case of boron.

Toble 2: $\mathrm{Si}(\ln )$ somples

\begin{tabular}{|c|c|c|c|c|c|c|}
\hline somple & $\alpha[\Omega \mathrm{cm}]$ & $\begin{array}{l}\text { concen } \\
\text { [ln] }\end{array}$ & $\begin{array}{l}\text { otions [1 } \\
\text { [0] }\end{array}$ & $\begin{array}{l}\left.{ }^{21} m^{-3}\right] \\
{[C]}\end{array}$ & $Y_{\text {max }}$ in & $\begin{array}{l}\mathrm{Hz} \text { from } \\
\text { (d) }\end{array}$ \\
\hline $\begin{array}{cc}S & 33 \\
\text { S } & 52 \\
S & 123 \\
\text { S } & 196\end{array}$ & $\begin{array}{l}2.0 \\
0.1 \\
1.29 \\
0.55\end{array}$ & $\begin{array}{l}500.0 \\
100.0\end{array}$ & $\begin{array}{l}700.0 \\
800.0 \\
<10.0\end{array}$ & $<1.0$ & 3.2 & $\begin{array}{l}14.5 \\
\approx 5\end{array}$ \\
\hline
\end{tabular}

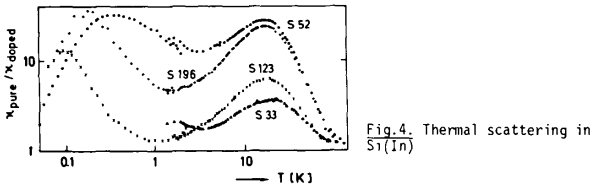

1 H.Zeile, K.Laßmann, Phys. Stat. Sol. (b) $111,555(82)$

2 A.M.de Goer, M.Locatelli and K.Laßmann, J. de phys. 42 C 6-235(81)

3 H.Zeile, U.Harten and K.Laßmanr, Phys. Stat. Sol. (b) $111,213(82)$

4 J.Maier and E.Sigmund, these Proceedings

5 S 52 obtained by J.S.Blakemore (sample 260 in PR B4 1873(71))

$S 123$ obtained by R.Helbig, Univ. Erlangen (sample Ru 237/1-1b) 